\title{
Aktuelle Studien zur Tanztherapie und Bewegungsanalyse bei Burnout, Williams-Syndrom und Parkinson
}

\author{
Iris Bräuninger
}

$\mathrm{D}$ ieser Beitrag präsentiert drei Studien zu unterschiedlichen Themen: Der erste Beitrag beschäftigt sich mit dem Potential der Bewegungsanalyse als Ergänzung im diagnostischen Prozess von Burnout (Pfeffer et al. 2018). Die Pilotstudie von Takahashi et al. (2020) widmet sich einem Interventionsbereich, der für die meisten TanztherapeutInnen unbekannt sein könnte: die Wirkung der Tanztherapie auf Stabilität, Muskelkraft und dynamisches Gleichgewicht bei Kindern mit Williams-Syndrom, einer seltenen Entwicklungsstörung. Der letzte Beitrag beschäftigt sich mit der Wirksamkeit der Tanztherapie bei Parkinson-Erkrankung auf Balance, Kognition und Wahrnehmung (Fisher et al. 2020).

\section{Zusammenhang zwischen Burnout und Bewegungsausdruck}

Pfeffer und KollegInnen (2018) gehen davon aus, dass ein Zusammenhang zwischen Burnout und Bewegungsausdruck besteht. Das erweiterte Wissen über das Bewegungsprofil von Burnout-PatientInnen könnte ihrer Meinung nach neue Erkenntnisse für die Untersuchung, Diagnose und Behandlung des Syndroms und dessen Prävention liefern. Ziel ihrer Studie war, das Bewegungsprofil und den Bewegungsausdruck hospitalisierter Burnout-
PatientInnen ( $n=22$; Alter 47.2; \pm 9.1 Jahre) anhand des LMA Effort Systems zu analysieren und mit dem von gesunden ProbantInnen ( $n=20$; Alter 41,5; \pm 15.0 Jahre) zu vergleichen. Alle Teilnehmenden füllten eine standardisierte Burnout-Inventarskala aus und nahmen an drei Bewegungsteilen teil, welche per Video aufgenommen wurden: 1. Warm up (10 min), 2. einfache Bewegungsinstruktionen (20 min), 3. Improvisationsteil zu den Themen Erde, Wasser, Feuer, Luft (20 min). Alle Videoaufnahmen wurden von zwei BewegungsanalytikerInnen (welche blind für die Gruppenzugehörigkeit der Teilnehmenden, das Studienziel und die Hypothese waren) anhand des LMA Effort Systems ausgewertet. Die Interrater-Übereinstimmung Kappa reicht von o,66 bis o,92 ( $p<0,001$ ), was als substantielle bis sehr gute Übereinstimmung gewertet werden kann. Die Ergebnisse zeigten, dass Burnout-PatientInnen im Vergleich zur Kontrollgruppe ein signifikantes Defizit in allen vier Antriebselementen aufwiesen: Sie zeigten weniger gebundene (Flow), indirekte (Space), leichte (Weight) und verzögerte Bewegungen (Time) und wiesen somit ein Defizit vor allem an genießenden Bewegungselementen auf. Die Elemente gebunden, indirekt, leicht und verzögert korrelierten mit Variablen von BOSS I und II und der Variablen Self von BOSS III. Diese Interkorrelation könnte als Anzeichen interpretiert werden, 
dass sich die Bewegungsanalyse als Ergänzung bei der Burnout-Diagnostik eignen könnte.

\section{Wirksamkeit der Tanztherapie bei Williams-Syndrom auf Stabilität, Muskelkraft und dynamisches Gleichgewicht}

Takahashi et al. (2020) untersuchten in einer explorativen Pilotstudie, ob Gruppen-Tanz-, Bewegungstherapie zur Verbesserung der Stabilität, des dynamischen Gleichgewichts, der Muskelkraft und der inneren Veränderungen beim Williams-Syndrom wirksam ist. Zwei Jungen und zwei Mädchen zwischen fünf und 10 Jahren nahmen an 10 TT-Gruppeninterventionen teil. Im Vor- und Nachtest wurden die Kinder mit statischen und dynamische BalanceFunktionstests und Muskelkrafttests geprüft. Ihre Eltern füllten zu beiden Untersuchungszeitpunkten die Child Behavioral Checkliste (CBCL) aus. Die TT-Gruppenintervention bestand aus den Teilen Warm-Up, Balanceübungen und Antriebselementen (Authentic Movement and LMA, S.67), Spiegeln mit Führen und Folgen von Bewegungsideen, Spielen mit einem Balanceball mit Griffen und Cool-DownPhase. Die Ergebnisse des statischen Funktionstests ergaben, dass sich das statische Gleichgewicht im Prä- / Post-Test-Vergleich verbesserte. Die Ergebnisse des Muskelkrafttests zeigten, dass drei von vier Teilnehmerlnnen einen Anstieg in Kniestreckung und alle eine Verbesserung der Beugungsmuskelkraft aufwiesen, jedoch kaum eine Verbesserung der Hüftbeugung. Beim dynamischen Gleichgewichtstest und beim $C B C L$ gab es keinen wesentlichen Unterschied vor und nach der TTIntervention.

\section{Wirksamkeit der Tanztherapie bei}

\section{Parkinson-Erkrankung auf Balance,} Kognition und Wahrnehmung

Fisher und KollegInnen (2020) überprüften in einer Interventionsstudie, ob sich die Gruppen-Tanztherapie bei der Parkinson-Erkrankung positiv auf Gleichgewicht, Kognition und Wahrnehmung auswirkt. Hierzu nahmen $11 \mathrm{Er}$ wachsene (sechs Frauen, Durchschnittsalter 67.8; fünf Männer, Durchschnittsalter 63.8) mit Parkinson im mittleren bis schweren Stadium an $10 \mathrm{TT}$-Gruppeninterventionen à $90 \mathrm{Mi}$ nuten mit dem Schwerpunkt Improvisationstanz (und der Möglichkeit individueller Pausen) teil. Zwei TanztherapeutInnen leiteten die Gruppe bilingual (englisch / französisch). Eine Person konnte nicht an den Terminen teilnehmen und wurde von der Datenanalyse ausgeschlossen. Die Stundenstruktur beinhaltete „Transition into the space“, Warm-Up, Bewegungsimprovisation zu einem vorgeschlagenen Thema, Führen-Folgen, Entwickeln einer Choreographie basierend auf Anliegen, Hoffnungen oder Bewegungselementen der Gruppenmitglieder, Reflexion der Bewegungserfahrung, Diskussion und Planung der nächsten Stunde. Das Ergebnis des Prä- / Post-Test-Vergleichs zeigt, dass Tanzbewegungstherapie mit Schwerpunkt Improvisationstanz zur funktionellen Verbesserung des Gleichgewichts und der Wahrnehmung beiträgt: Es wurde sowohl eine signifikante Verbesserung der Gesamtmotorfunktion $(t=2.39, p=<0.0001$, PräTest $=79.1 \pm 2.88 \%$ vs. Post-Test $=92.1 \pm 2.18 \%$ ) beim Balance Evaluation Systems Test als auch eine signifikante Verbesserung der Kognition $(t=2.575, p=0.0299$, Prä-Test $=56.7 \pm 3.73 \%$ vs. Post-Test $=62.2 \pm 4.43 \%$ ) beim Montreal Cognitive Assessment, vor allem bei der Exekutivfunktionsaufgabe, nachgewiesen. 


\section{Zusammenfassung}

Die hier vorgestellten Studien demonstrieren, dass sich die Tanz-, Bewegungstherapie nicht mehr nur auf den klassischen Bereich (psychiatrischer Kontext) beschränkt, sondern längst in anderen Bereichen Fuß gefasst hat und mit empirischen Studien ihre Legitimation zu belegen sucht.

\section{Literatur}

Fisher, M., Kuhlmann, N., Moulin, H., Sack, J., Lazuk, T., Gold, I. (2020): Effects of improvisational dance movement therapy on balance and cognition in Parkinson's disease. Physical \& Occupational Therapy In Geriatrics, 1-15, https://doi.org/10.1080/02703181.2020.176 5943

Pfeffer, M. M., Paletta, A., Suchar, G. (2018): New perspectives on Burnout: A controlled study on movement analysis of Burnout patients. Frontiers in Psychology 9, 1150, https://doi. org/10.3389/fpsyg.2018.01150
Takahashi, H., Seki, M., Matsumura, T., An, M., Sasai, T., Ogawa, Y., Matsushima, K., Tabata, A., Kato, T. (2020): The effectiveness of dance / movement therapy in children with Williams syndrome: A pilot study. American Journal of Dance Therapy 42 (1), 33-6o, https://doi. org/10.1007/s10465-020-09324-9

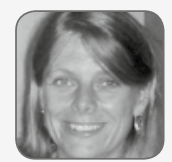

Dr. Iris Bräuninger

Senior Researcher \& Co-Leiterin Studiengang Psychomotoriktherapie (Hochschule für Heilpädagogik Zürich IVE),

Dozentin MA Tanztherapie UAB Barcelona, BTD-Supervisorin / Ausbilderin / Lehrtherapeutin, KMP-Notatorin, Praxis Tanztherapie Supervision Bodensee.

DDr. Iris Bräuninger dancetherapy@mac.com und iris.braeuninger@hfh.ch 